\section{Modelos contemporáneos de supervisión clínica: nuevas direcciones}

\section{Contemporary Models of Clinical Supervision: new Directions}

\author{
Ana Lilia Villafuerte Montiel \\ Universidad Iberoamericana \\ ana.villafuerte@ibero.mx \\ https://orcid.org/0000-0002-4772-065X
}

DOI: $10.19136 / \mathrm{mhr} . \mathrm{a} 5 \mathrm{n} 1.4210$

\begin{abstract}
Resumen
La supervisión clínica es una actividad profesional diseñada para formar psicoterapeutas competentes y éticos, cuyas intervenciones sean eficaces para brindar un servicio de alta calidad a los pacientes. Cumple con funciones formativas, restaurativas y normativas, las cuales dependen en buena medida del modelo de supervisión que se emplee. En este artículo se presenta una revisión de la literatura para dar cuenta de las categorías de modelos existentes y se proporciona una síntesis de algunos modelos comúnmente empleados en la supervisión. Se consultaron bases académicas pertenecientes a EBSCO, especificamente Psychology \& Behavioral Sciences Collection, así como ERIC, PsycArticles y Google Scholar, y se seleccionaron artículos que comprendidos entre 1995 y 2020 para llevar a cabo este análisis. La consulta empleó palabras clave en inglés: "clinical supervision", "supervision", "competence", "competency-based", "evidencebased supervision" y "psychotherapy-based supervision". Se presentan cuatro categorías de modelos, tres de las cuales son globalmente aceptadas y la cuarta se ofrece como una propuesta a integrar porque cuenta con características específicas y representa la evolución que ha tenido el área y está sustentada por la investigación.
\end{abstract}

\section{Palabras clave:}

modelos de supervisión, supervisión clínica, basados en psicoterapia, supervisión del desarrollo, modelos de proceso, basados en evidencia, factores comunes, psicoterapia

\footnotetext{
Abstract

Clinical supervision is a professional activity designed to train competent and ethical psychotherapists who can provide effective and high-quality interventions to the clients. It fulfills educational, restorative and normative functions, which largely depend on the supervision model used. This article presents a review of the literature to account for the categories of existing models and provides a synthesis of some models commonly used in supervision. EBSCO academic databases were consulted, especifically Psychology \& Behavioral Sciences Collection and ERIC, also PsycArticles and Google Scholar were used; the articles selected ranged from 1995 to 2020. Only keywords in English were used: "clinical
} 
supervision", "supervision", "competence", "competency-based", "evidence-based supervision" and "psychotherapy-based supervision". Four categories of models are presented, three of which are globally accepted and the fourth is offered as a proposal to be integrated since it has specific characteristics and represents the evolution of the area, which is supported by research.

\section{Keywords:}

models of supervision, clinical supervision, psychotherapy-based, development supervision, process models, evidence-based, common factors, psychotherapy

La supervisión clínica es un espacio educativo, de reflexión y de desarrollo técnico que permite a las y los psicoterapeutas, de cualquier modelo teórico, adquirir experiencia profesional de manera segura y sistemática. Se trata de una piedra angular en la formación de las y los profesionales de la salud mental porque proporciona habilidades, valores, actitudes, estrategias de intervención y nociones teóricas que favorecen la eficacia de los tratamientos (Falender \& Shafranske, 2004; Bernard \& Goodyear, 2014; Holloway \& Neufeldt, 1995; Ladany et al., 2013; O'Donovan et al., 2011). Es una relación que se caracteriza por ser evaluativa y jerárquica porque busca desarrollar y perfeccionar competencias clínicas y es brindada por un profesional experimentado hacia uno principiante (Corey et al., 2010; Milne, 2007; Watkins, 2020), con la finalidad de salvaguardar el bienestar del paciente. De esta manera, la supervisión cumple tres funciones: formativa, restaurativa y normativa. La función formativa apunta a facilitar el aprendizaje y la pericia del supervisado; la restaurativa fomenta el procesamiento emocional de los casos; y la normativa, provee un estándar de calidad en la disciplina. Finalmente, la supervisión apoya el análisis y resolución de dilemas éticos que pudieran presentarse en los procesos de ayuda. Es un área de especialización en sí misma (American Psychological Association [APA], 2014; Norcross \& Popple, 2017) por lo que requiere entrenamiento y capacitación específicos.

Cuando se va a proveer supervisión es necesario formalizar la relación a través de un contrato (Borders, et al., 2014; Hawkins \& McMahon, 2020) en el cual se especifican aspectos relevantes, tales como: el formato, el modelo bajo el cual el supervisor va a conducirla, las metas, las expectativas de ambas partes, la normatividad aplicable, el método de evaluación y los costos, entre otros temas. La intención del presente artículo es precisamente hacer una revisión general de los modelos de supervisión existentes, ya que son el componente esencial para orientar dicho proceso debido a que ofrecen una estructura lógica y dan congruencia a las actividades que se realizarán. Estar familiarizado con los diversos modelos favorece una práctica actualizada, ética y plural que tiene un impacto directo en la calidad y eficiencia de los servicios psicoterapéuticos que se brindan a la población. Esta revisión proporcionará al lector un panorama amplio sobre la multiplicidad de opciones que existen, identificará cómo han ido evolucionando y reflexionará la manera en que se pueden integrar en la práctica profesional para enriquecerla.

\section{Método}

Se consultaron bases académicas pertenecientes a EBSCO, específicamente Psychology \& Behavioral Sciences Collection, así como ERIC, PsycArticles y Google Scholar. Se seleccionaron artículos que comprendidos entre 1995 y 2020 con las palabras clave en inglés: "clinical supervision", "supervision", "competence", "competency-based", "evidencebased supervision" y "psychotherapy-based supervision". También se consultaron textos clásicos que apoyaran la comprensión de los contenidos a exponer y analizar.

\section{Identificación de Modelos}

De manera análoga a la psicoterapia, la supervisión es una actividad compleja que puede partir de preceptos teóricos muy diversos, razón por la cual, no existe un solo modelo que dé cuenta de todas las dinámicas y los aspectos a examinar dentro de la supervisión (Bernard \& Goodyear, 2014; McNeill \& Stoltenberg, 2016; Norcross \& Popple, 2017). Por otra parte, la supervisión es un área de especialización que ha ido transformándose en el tiempo de acuerdo 
con el contexto, el momento histórico y a las necesidades tanto de los supervisados como de la población a la que se atiende. Con base en la revisión de la literatura, se tiene identificado que hay tres categorías generales de modelos de supervisión: los que se fundamentan en corrientes psicoterapéuticas, los que parten de las etapas de desarrollo del supervisado y los que se centran en el proceso mismo de la supervisión. No obstante, en este artículo se propone ya considerar una cuarta categoría que se sustenta en factores comunes (Morgan \& Sprenkle, 2007), en la investigación en el campo de la supervisión y en modelos multiteóricos. Algunos autores (Bernard \& Goodyear, 2014; Hawkins \& McMhon, 2020; Watkins \& Milne, 2014) no consideran que sea una categoría nueva porque varias de las propuestas surgen de la combinación de modelos preexistentes, sin embargo, también se han creado posturas para cubrir necesidades específicas de la supervisión, las cuales generalmente están basadas en evidencias (David, Lynn \& Montgomery, 2018; Falender \& Shafranske, 2010).

En la Figura 1, de acuerdo con la presente revisión, se muestran las principales categorías de supervisión y algunos los modelos generales contenidos en ellas. Cabe aclarar que de ninguna manera abarcan el universo de opciones disponibles. Es necesario mencionar que, en la práctica, los supervisores no ejercen la supervisión "dentro" de estas categorías de manera exclusiva sino "entre" ellas (Bernard \& Goodyear, 2014; McNeill \& Stoltenberg, 2016; Milne \& Reiser, 2012; Norcross \& Wampold, 2018). Este argumento nace porque la mayoría de profesionales en supervisión están entrenados en una corriente psicológica y de ella parten para estructurar el proceso de supervisión. Cuando se realiza la supervisión de manera cuidadosa y responsable, también deben considerarse factores como el nivel de desarrollo del supervisado y el proceso mismo de la supervisión.

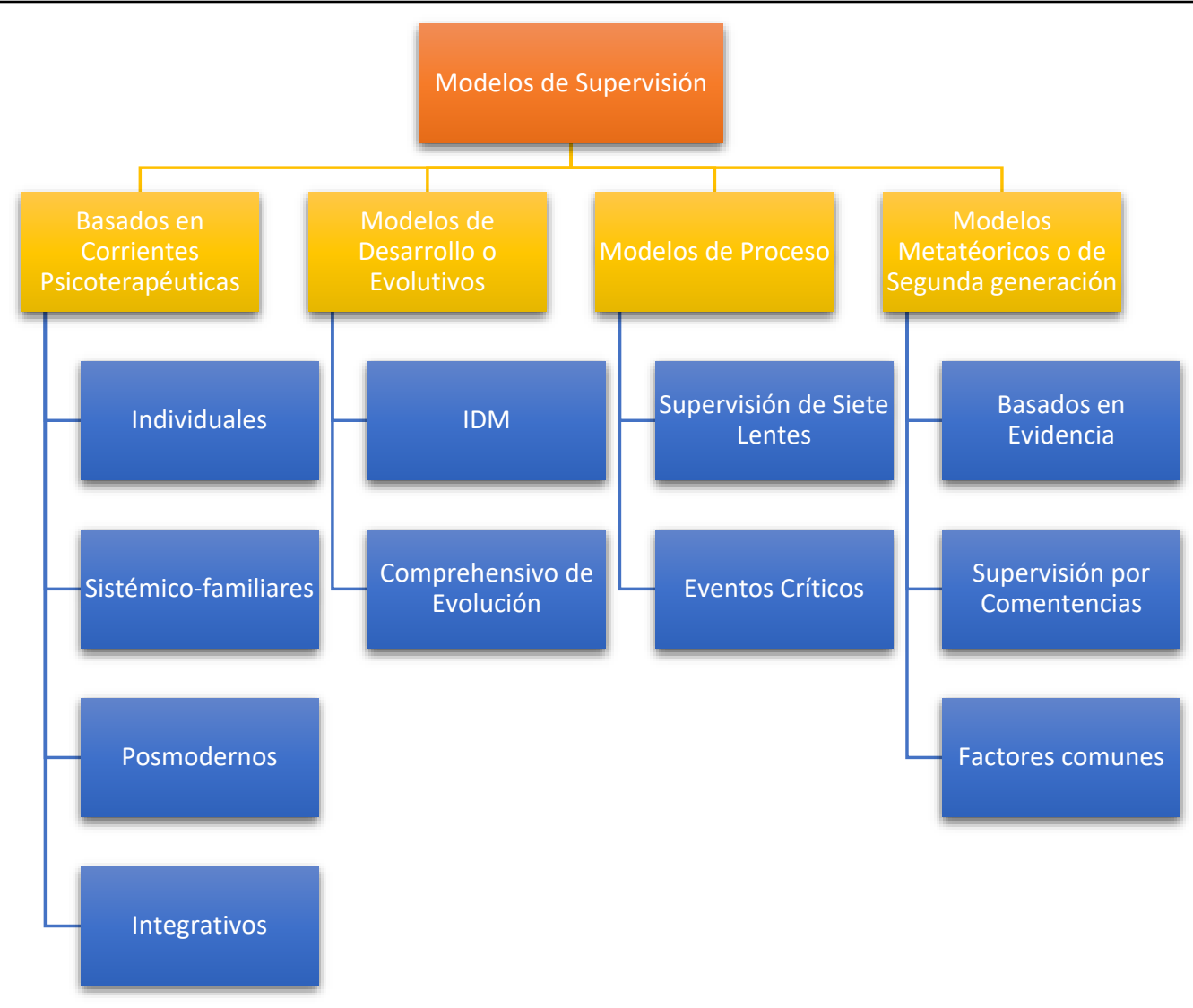

Fig.1 Principales categorías de modelos de supervisión 
Pareciera que de forma natural los supervisores intersecan las categorías para realizar eficazmente su trabajo.

\section{Basados en corrientes psicoterapéuticas}

La supervisión nació de la mano de los primeros modelos psicoterapéuticos, en particular, con el Psicoanálisis. De acuerdo con Eagle y Long (2014), Freud fue el primer supervisor en psicoterapia, ya que desde 1902, sostenía reuniones periódicas en su casa para discutir los casos con sus colegas y él decidía cómo debían manejarse los procesos. De manera similar, los creadores de modelos subsecuentes fueron los que también promovieron la supervisión apegados a sus estándares y preceptos rectores. Este tipo de supervisión tiene varias ventajas, las dos principales son:

- Es un entorno de aprendizaje isomórfico (Pearson, 2006) porque el paradigma y las estrategias que promueven el cambio en terapia, son las mismas que favorecen el cambio en el supervisado.

- Permite hacer un modelamiento del establecimiento de la alianza de trabajo en supervisión y trasladarlo a la psicoterapia (Bradley \& Gould, 2001).

Sus principales desventajas son:

- Puede privilegiar las habilidades de intervención por encima del progreso del paciente y/o de las necesidades de capacitación de la supervisada o del supervisado. Incluso podría bloquear la apertura a otros abordajes al mostrar el modelo que se está aprendiendo como superior a otros paradigmas (Pearson, 2006). Por otro lado, se señala que este estilo de supervisión pudiera nublar u obstaculizar la mirada hacia otras propuestas psicoterapéuticas viables que pudieran parecer "incompatibles" con la corriente empleada (Pearson, 2006).

- La supervisión, en términos generales, no ha evolucionado debido a que tampoco los modelos de los que parten lo han hecho a pesar de las transformaciones socioculturales y contextuales (Watkins, 1995).

Dentro de esta categoría se ubican la supervisión de modelos individuales, sistémicos-familiares, posmodernos e integrativos.

\section{Modelos individuales}

Las características principales de los modelos individuales es que parten de la concepción de que el cambio parte del individuo y no hace tanto énfasis en el contexto, las condiciones intervinientes o los sistemas en los que está inmersa la persona (JonesSmith, 2016). En esta sección hablaremos de la supervisión psicoanalítica, cognitivo-conductual y humanista- existencial.

\subsection{Supervisión en la terapia psicoanalítica}

Dado que fue el primer modelo psicoterapéutico que se creó, su estilo de supervisión empezó a permear sin sistematización, hasta que Max Eitingon, quien presidió la Asociación Psicoanalítica Internacional propuso el Modelo Eitingon para entrenamiento de analistas en formación (Schwartz, 2015). Este modelo se sigue empleando en diferentes partes del mundo, pero ahora con variaciones. Posteriormente Ekstein y Wallerstein (1972) articularon una propuesta de supervisión que fue bien recibida por analistas de varias escuelas. Ellos cambiaron la perspectiva para que no se centrara exclusivamente en el paciente y sus dinámicas, sino en el aprendizaje, desarrollo y dinámicas del supervisado (Falender \& Shafranske, 2004; Frawley-O'dea y Sarnat, 2001), convirtiéndose en un modelo más relacional. De aquí se originó el Proceso Paralelo, herramienta que se enfoca en analizar lo que sucede al terapeuta con su paciente, pero también lo que pasa en la relación entre el terapeuta y el supervisor (Bernard \& Goodyear, 2014). El objetivo final es que el supervisado comprenda la dinámica de resolución de conflictos relacionales (Eagle \& Long, 2014) que se dan entre el supervisor(a) y él o ella, para que posteriormente lo pueda aplicar con sus futuros pacientes.

\subsection{Supervisión en las terapias cognitivas y conductuales (TCC)}

Se catalogan como terapias cognitivo- conductuales todas aquellas que parten de premisas de la modificación de la conducta mediante condicionamiento y la modificación del pensamiento. Cabe destacar que los modelos TCC son los que poseen mayor sustento empírico con respecto a su eficacia, ya que están respaldados por ensayos clínicos extensos que cubren una amplia variedad de grupos de diagnóstico y problemas psicológicos (Butler, et al., 2006; Pretorius, 2006; Reiser \& Milne, 2012). De manera similar, su propuesta de supervisión posee fundamento científico y es un 
proceso sistematizado postulado por Liese y Beck (1997) y posteriormente actualizado por Newman (2010). La supervisión es proceso basado en evidencia, es pragmático, colaborativo y su meta es perfeccionar las habilidades de conceptualización e intervención del supervisado (Milne, 2007).

\subsection{Supervisión de modelos humanistas y existenciales}

Estos modelos se integran en uno solo porque comparten principios rectores y la persona es considerada como una entidad biopsicosocial, indivisible y que debe conocerse fenomenológicamente. En ese sentido, la experiencia humana consciente y su potencial, así como la libertad, son centrales en este modelo (Bernard \& Goodyear, 2014; Farber, 2010). De este modo es que la supervisión es un acompañamiento para que el supervisado incremente su capacidad de autoexploración y su habilidad para fungir como agente de cambio (Farber 2010; Pack, 2009). Roth y Pilling (2015), consideran como una competencia indispensable el aprender a manejar el self para estar con atención plena con el paciente, para mostrarse genuino, respetuoso y transparente.

\section{Modelos Sistémico-Familiares y de Pareja}

De acuerdo con Rigazzio-DiGilio (2014) lo que caracteriza a este modelo es que elabora los casos desde una comprensión integral de la persona, el contexto y las dinámicas interpersonales, lo cual favorece organizar y analizar varios sistemas simultáneamente (Falender \& Shafranske, 2004). Este modelo integra las construcciones culturales, el estilo de comunicación, el apego y los contextos (educativo, social, económico), para bordar alrededor de las necesidades de la persona o la familia (Simon, 2010; Todd \& Storm, 2014) y ofrecer opciones que conduzcan a una solución. Esta propuesta considera que tanto los terapeutas como los supervisores son agentes de cambio, que "pertenecen" al sistema y pueden incidir en él (Simon, 2010; Roth \& Pilling, 2015). La supervisión puede ser tener varios formatos: desde lo individual, pasando por la supervisión en vivo con o sin equipo reflexivo, en coterapia o bien, grupal. Estas variantes aumentan el nivel de complejidad, ya que se amplían los sistemas y las dinámicas que se pueden presentar.

\section{Supervisión de Modelos Posmodernos}

La supervisión colaborativa y narrativa están consideradas dentro de esta subcategoría porque comparten una base epistemológica socioconstruccionista y ambas se fundamentan en el posmodernismo y en la hermenéutica. La posmodernidad es una crítica ideológica a la tradición moderna de que las narrativas representan verdades universales y de que las cosas existen en sí (Anderson \& Swim, 1995). En otra palabras la postura posmoderna sostiene que hay una multiplicidad de perspectivas, de realidades humanas o "voces" y que no hay una verdad absoluta (Gergen, 1997; Tarragona 1999). Harlene Anderson y Harry Goolishian dieron origen a la Terapia Colaborativa, que más que una escuela teórica se trata de una forma de ser y estar con las personas, coconstruyendo la realidad a partir de una postura de "no conocer" (not- knowing position) y de la posibilidad de generar diversas perspectivas para construir nuevos significados (Anderson \& Swim, 1995; Monk \& Gehart, 2003). Por su parte, la terapia narrativa surge del trabajo de Michael White y David Epston, sus ideas centrales están relacionados con que las historias determinan el significado de la experiencia y que la deconstrucción, como herramienta, permite visualizar la realidad desde una narrativa constructiva y que promueva el cambio (Monk \& Gehart, 2003; White, 1997). La supervisión está permeada por todos estos preceptos, donde la voz de los supervisados cobra un cariz particular y en la cual se emplean estrategias para apoyar voces distintas a la propia, como lo es el Equipo Reflexivo (Andersen, 1994). La jerarquía es totalmente horizontal y cada sesión constituye un encuentro entre dos expertos. En la supervisión se enfatiza que el lenguaje tiene un lugar preponderante, ya que construye realidades sociales y genera conversaciones significativas que facilitan intercambios sociales (Hoffman, 1999; McNamee \& Gergen, 1999).

\section{Supervisión de Modelos Integrativos}

Este modelo psicoterapéutico es casi tan antiguo como los modelos individuales, pero no había tenido tanta atención sino hacia la década de 1960 cuando la investigación en psicoterapia empezó a tener más presencia en los medios académicos (Jones-Smith, 2016). Los estudios mostraban que no había diferencias significativas en la eficacia y eficiencia de los modelos entre sí, sino que había elementos que 
cortaban a través de ellos como la alianza terapéutica o los factores extra-terapéuticos (Miller et al., 2004). Con esa inercia se gestó el interés por agrupar los modelos bajo un mismo marco epistemológico, llevando el conocimiento hacia una arena traslacional. Derivado de este abordaje surgen varios caminos para la integración de modelos (Norcross \& Wampold, 2018; Norcross \& Popple, 2017) a saber: el eclecticismo técnico, la integración teórica, la integración asimilativa o selectiva y la integración de factores comunes.

El objetivo de la supervisión integrativa es aprovechar las diversas orientaciones teóricas y la evidencia en investigación para perfeccionar las habilidades clínicas de los supervisados (Bernard \& Goodyear, 2014) y que esto se vea reflejado en los logros de los pacientes. Al igual que en la TCC, la supervisión es un proceso sistemático e informado por la investigación que busca realizar una evaluación transdiagnóstica, que oriente el diseño de la intervención.

\section{Modelos Evolutivos de la Supervisión}

Estos abordajes parten de la idea de que los profesionales de la salud mental atraviesan por distintas etapas en su desarrollo profesional (Salvador, 2016; Skovholt \& Rønnestad, 2003; Vethencourt D'Escriva, 2018) que se deben identificar para ajustar el proceso de supervisión a la medida de las necesidades de capacitación. La ventaja de esta categoría es que es compatible con cualquier escuela teórica, ya que se centran en las actitudes, habilidades y conocimientos característicos desde el nivel de principiante hasta el de experto (Falender \& Shafranske, 2004; Stoltenberg et al., 2014). Todas las propuestas parten de diferentes teorías del desarrollo, por lo tanto, su nivel de complejidad varía.

\section{Modelo Comprehensivo de Desarrollo de Loganbill, Hardy y Delworth}

Esta propuesta de supervisión se fundamenta en las tareas de desarrollo de la juventud de Chickering (Bernard \& Goodyear, 2014), pero las reconceptualiza para ser aplicadas al entrenamiento de psicoterapeutas. Las áreas para observar son: la competencia, la autonomía, la autoconsciencia emocional, la motivación personal, la identidad profesional, el respeto por las diferencias individuales, el propósito y dirección, asi como la ética profesional. La persona en supervisión puede situarse en alguna de las tres etapas: estancamiento, confusión o integración (Loganbill et al.,1982), o bien, que esté en transición entre estas. De manera somera, las etapas se describen de la siguiente manera:

- Estancamiento: se aprecia que no hay progreso en las habilidades del supervisado 0 supervisada, ya sea por falta de conocimiento 0 por incapacidad para observar las propias deficiencias o dificultades.

- Confusión: esta etapa puede tener un inicio gradual 0 abrupto. El 0 la profesional en supervisión muestra inestabilidad y desorganización, con fluctuaciones erráticas, confusión y sensación de estar en conflicto con algo 0 alguien.

- Integración: hay una evolución donde se presenta un ajuste cognitivo que promueve la autoeficacia, la seguridad del supervisado 0 supervisada y la flexibilidad de pensamiento.

\section{Modelo Integrado de Desarrollo (IDM) de Stoltenberg}

El Modelo Integrado de Desarrollo (IDM, por sus siglas en inglés) propone identificar la etapa en la que se encuentra el supervisado para guiar al supervisor con respecto al tipo de apoyo que éste requiere (McNeill \& Stoltenberg, 2016). El modelo posee una estructura compleja y comprehensiva a fin de ofrecer un marco de referencia amplio que abarque los diferentes estilos de aprendizaje de los supervisados (McNeill \& Stoltenberg, 2016; Salvador 2016). Stoltenberg describe cuatro etapas de desarrollo en las cuales se deben fomentar habilidades y competencias específicas en el supervisado y al supervisor o supervisora, se le provee también de una serie de estrategias para poder cumplir con esta tarea (Corey, et al., 2010). Para la identificación de la etapa, se parte de una matriz creada por las intersecciones entre las estructuras primordiales (competencias específicas) y por el nivel de progreso y experiencia de las y los profesionales en supervisión. Las estructuras primordiales son la motivación, la autonomía y la consciencia de sí mismo y de otros. Los niveles son desde el de (1) principiante, (2) intermedio, (3) avanzado y un subnivel (3i) que se distingue por ofrecer una mayor consolidación de habilidades y de conocimientos, así como una identidad profesional más definida. 


\section{Modelos de Proceso o del Rol Social}

La supervisión, como proceso, tiene el papel central en las propuestas de esta categoría porque se trabaja en ella desde lo relacional y lo educativo (Bernard \& Goodyear, 2014; Falender \& Shafranske, 2004). Su complejidad es variada dependiendo de aquello que se pretende describir dentro de la supervisión y del tamaño del sistema o sistemas que se involucren en el proceso.

\section{Modelo de Supervisión Basado en Eventos Críticos}

Esta propuesta destaca porque Ladany, Friedlander y Nelson (2005) se fundamentan en la práctica sustentada empíricamente. Aunque es un modelo que no está anclado con ninguna corriente teórica, muestra atisbos de modelos interpersonales, humanistas y psicoanalíticos actuales (Friedlander, 2015; Sarnat, 2010). La premisa rectora es que todos los involucrados (supervisados, supervisores y pacientes/clientes) son participantes activos en la comprensión de los procesos de la supervisión y la terapia (Ladany et al., 2016). Se trabaja con situaciones que representan un dilema o un impasse, ya sea dentro de la propia relación de supervisión 0 en el trabajo del supervisado con uno o más de sus pacientes. De acuerdo con Grant, Schofield y Crawford (2012) este modelo promueve la competencia del supervisado, pero desde un marco estructurado para que el supervisor sepa cómo resolver situaciones problemáticas que generalmente no están descritas en manuales o que no son abordadas en entrenamientos.

Cuando surge una situación que genera conflicto en la supervisión o en la terapia, ya sea que el supervisado lo mencione o que el supervisor lo haga evidente, se habla de que hay un "marcador" (Bernard \& Goodyear, 2014). Dicho marcador será elaborado desde un entorno de tarea, es decir, mediante diversas interacciones entre el supervisor y el supervisado (Ladany et. al, 2005, 2016) para comprender el conflicto, entender la dinámica subyacente y explorar las mejores opciones de solución. Una vez que se llega a la comprensión del problema y a soluciones viables, se procede a ponerlo en práctica.

\section{El Modelo de Siete Lentes de Hawkins y Shohet}

Este tipo de supervisión se distingue porque abarca no sólo la diada supervisora, sino los contextos organizacionales y sociales (Bernard \& Goodyear, 2014; Hawkins \& McMahon, 2020). Se sustenta en varias escuelas psicológicas, que van desde los enfoques individuales como el psicoanálisis y el cognitivo-conductual hasta posturas sistémicas. También se complementa con teorías del aprendizaje y del desarrollo de adultos, las neurociencias y la teoría del cambio. Dada su naturaleza, este modelo de supervisión no es privativo de la psicoterapia, sino de otras profesiones que establezcan relaciones de ayuda, como en enfermería, derecho y trabajo social. El proceso de supervisión involucra una doble matriz (Hawkins 1985; Hawkins \& Shohet, 2006), conformada por (1) paciente-supervisado(a) y (2) supervisado(a)-supervisor(a). Dichas matrices existen dentro de contextos o sistemas más amplios que inciden en ellas y tienen el poder de alterarlas. De las posibles combinaciones de estas matrices con otros elementos se pueden identificar siete lentes 0 modos para realizar la supervisión, que son:

1) Modo 1: La atención se concentra en los detalles del caso y del paciente.

2) Modo 2: centrarse en las estrategias e intervenciones del supervisado o supervisada.

3) Modo 3: enfocarse en la relación entre el cliente/paciente y el supervisado.

4) Modo 4: atención en el supervisado. Se revisa cómo el profesional en supervisión se ve afectado(a) consciente e inconscientemente por el trabajo con sus pacientes o clientes (Hawkins \& McMahon, 2020).

5) Modo 5: centrarse en la relación de supervisión.

6) Modo 6: el supervisor o la supervisora se centra en su propia experiencia al escuchar a la persona en supervisión en el "aquí y ahora".

7) Modo 7: la atención se centra en los contextos más amplios en los que se desarrolla el trabajo terapéutico.

La meta de supervisión es acompañar a profesionales en formación de acuerdo con la perspectiva que requiera, ya sea que se trate de facilitar su insight, elaborar el caso o bien, aprender a trabajar desde la realidad del paciente y de los sistemas en los que está inmerso.

\section{Modelos Metateóricos o de Segunda Generación}

Esta es la cuarta categoría que se propone añadir a las tres globalmente aceptadas: basadas en un modelo terapéutico, centradas en el desarrollo y las del proceso de la supervisión. Esta es una 
clasificación con características específicas y si bien comprende modelos que surgen de la combinación integrada de otros, también ofrece propuestas novedosas sustentadas en la evidencia y pondera el empleo de múltiples teorías (transteóricos y multiteóricos) para generar un marco de referencia más inclusivo. Es importante recordar que los avances de investigación en psicoterapia y las actualizaciones en las corrientes terapéuticas han hecho que el campo de la supervisión también se transforme (Milne, et al., 2008). Asimismo, debe evolucionar nuestra comprensión de los categorías que existen. A continuación, se describen de manera general dos de estas propuestas.

\section{Modelos de factores comunes}

Es el modelo psicoterapéutico y de supervisión que mayor evidencia científica posee. Una de las propuestas es trabajo intelectual de Morgan y Sprenkle (2007), quienes realizaron una revisión sistemática de los modelos de supervisión en la literatura en salud mental e identificaron diversos dominios que son comunes a varios de estos. Por otro lado, también categorizaron actividades comunes en supervisión en 48 clases. Los dominios están relacionados con las habilidades, las estrategias, el conocimiento, los valores, la ética y la evaluación, entre otros temas relevantes. Morgan y Sprenkle (2007) identificaron tres constructos, llamados continuos, que contienen una amplia variabilidad de los dominios y actividades del modelo:

- Énfasis: los extremos de este constructo son la competencia profesional y la competencia clínica.

- Especificidad: los polos van de lo idiosincrásico o particular a lo nomotético o general

- Relación: que se caracteriza por ir desde lo colaborativo y lo totalmente directivo.

De las posibles combinaciones, pudieron derivar cuatro roles principales ejercidos por profesionales de la supervisión: profesor(a), mentor(a), coach y administrador(a).

\section{Supervisión por competencias}

Toda vez que la supervisión clínica es una práctica profesional distinta a la psicoterapia, surge la necesidad de un entrenamiento formal $y$ sistematizado para enseñar sus competencias a los supervisores (Falender \& Shafranske, 2004, 2010). Se define como "competente" a aquella persona calificada y altamente entrenada para desempeñar una función profesional específica de manera eficaz (Kaslow, et al., 2007). La competencia hace referencia a un conjunto de conocimientos, habilidades, actitudes, valores y ética requeridos en los servicios de salud mental, que es el objetivo de entrenamiento y supervisión clínica (Falender et al., 2014; Falender \& Shafranske, 2010).

No obstante, es relevante señalar que la competencia es un estado de suficiencia en relación con la capacitación requerida dentro de un entorno determinado (Falender \& Shafranske, 2004). Es decir, es un constructo relacional que depende de las demandas del escenario o del contexto, por lo tanto, los y las profesionales de la salud mental siempre pueden seguir aprendiendo y perfeccionando sus conocimientos, al igual que los profesionales de la supervisión. Todo modelo de supervisión promueve la competencia, la distinción con esa propuesta es que proporciona una base teórica sólida y un método explícitos para introducir, desarrollar, implementar y evaluar dichas competencias en la supervisión (Falender \& Shafranske, 2004). Promueve la aplicación del conocimiento y las habilidades en el mundo real y utiliza los resultados del desempeño como criterios para evaluar tanto a los alumnos como a los programas de capacitación.

\section{Conclusiones}

Como lo muestra presente revisión, existe una gran diversidad en modelos de supervisión y todos suman a la experiencia de esta actividad. Familiarizarse con ellos aumenta la riqueza, la calidad y las perspectivas bajo las cuales se realiza la supervisión y, por tanto, contribuye a potencializar tratamientos psicoterapéuticos (Wheeler \& Richards, 2007). Por otro lado, emplear diferentes propuestas ayuda a promover prácticas éticas y eficientes porque el supervisor puede responder a las necesidades del supervisado o del caso de una manera más fluida y estructurada, sin dejar lugar a la improvisación. Si a través del modelo de supervisión empleado se puede generar un espacio de contención y de colaboración, la supervisión será personalizada, gratificante y formará profesionales de la salud mental comprometidos con su labor (Villafuerte, 2016). Adicionalmente, es imprescindible descartar la idea de la supremacía de un modelo sobre otro y contemplar que son aproximaciones que de ninguna manera son mutuamente excluyentes, sino que se complementan entre sí y que permiten al supervisor 0 supervisora tener una mirada panorámica sobre las 
dinámicas al interior de la psicoterapia y de la supervisión.

Finalmente, a lo largo del artículo se aprecia la evolución de los modelos y cómo se han ido haciendo cada vez más complejos, exhaustivos y enfocados en estrategias que realmente facilitan el abordaje de situaciones conflictivas en supervisión. La pregunta para reflexionar sería: ¿podemos los y las profesionales de la supervisión también transformar nuestra práctica y ampliar nuestras competencias?

\section{Referencias}

American Psychological Association. (2014a). Guidelines for Clinical Supervision in Health Service Psychology. http://apa.org./about/policy/guidelinessupervision.pdf

Andersen, T. (1994). El equipo reflexivo: diálogos y diálogos sobre los diálogos. México: Gedisa.

Anderson, H \& Swim, S. (1995). Supervision as a Collaborative Conversation: Connecting the voices of a supervisor and supervisee. Journal of Systemic Therapies, Vol.14, pp 1-13. https://doi.org/10.1521/jsyt.1995.14.2.1

Bernard, J. \& Goodyear, R. (2014). Fundamentals of clinical supervision $\left(5^{\text {th }}\right.$. Ed.). Estados Unidos: Pearson Education.

Borders, L.D., Glosoff, H.L., Welfare, L.E., Hays, D.G., DeKruyf, L., Fernando, D.M. \& Page, B. (2014). Best Practices in Clinical Supervision: Evolution of a Counseling Specialty. Faculty Publications - Graduate School of CounselingPaper

7.

https://digitalcommons.georgefox.edu/cgi/viewcont ent.cgi?article $=1007 \&$ context $=$ gsc

Bradley, L. J., \& Gould, L. J. (2001). Psychotherapybased models of counselor supervision. In L. J. Bradley \& N. Ladany (Eds.), Counselor supervision: Principles, process, and practice (3rd ed., pp. 147180). Filadelfia: Brunner-Routledge.

Butler, A. C., Chapman, J. E., Forman, E. M., \& Beck, A. T. (2006). The empirical status of cognitivebehavioral therapy: A review of meta-analyses. Clinical Psychology Review, 26, 17-31. https://doi.org/10.1016/j.cpr.2005.07.003

Corey, G., Haynes, R., Moulton, P. \& Muratori, M. (2010). Clinical supervision in the helping professions: a practical guide ( $2^{\text {nd }}$ ed.). Estados Unidos: American Psychological Association.

David, D., Lynn, S.J. \& Montgomery, G.H. (2018). Evidence-based psychotherapy: The state of the science and practice. Hoboken, NJ: John Wiley \& Sons, Inc.

Eagle, G. \& Long, C. (2014). Supervision of the Psychoanalytic/Psychodynamic Psychotherapy. En Watkins, E., \& Milne, D. (Eds.). The Wiley International handbook of clinical supervision (pp. 471-492). Reino Unido: John Wiley \& Sons, Ltd.

Ekstein, R., \& Wallerstein, R. (1972). The teaching and learning of psychotherapy (2da. Ed.). Nueva York: International Universities Press, Inc. https://psycnet.apa.org/record/1973-26882-000

Falender, C. \& Shafranske, E. (2004). Clinical supervision: a competency-based approach. Estados Unidos: American Psychological Association.

Falender, C. A., \& Shafranske, E. P. (2010). Psychotherapy-based supervision models in an emerging competency-based era: a commentary. Psychotherapy (Chicago, III.), 47(1), 45-50. https://doi.org/10.1037/a0018873

Falender, C.A., Shafranske, E.P. \& Ofek, A. (2014). Competent clinical supervision: Emerging effective practices. Counselling Psychology Quarterly, 27 (4), 393-408.

https://doi.org/10.1080/09515070.2014.934785

Farber, E. W. (2010). Humanistic-existential psychotherapy competencies and the supervisory process. Psychotherapy Theory, Research, Practice, Training, 47, 28-34. https://psycnet.apa.org/doi/10.1037/a0018847

Frawley-O'Dea, M. G., \& Sarnat, J. E. (2001). The supervisory relationship: A contemporary psychodynamic approach. New York: Guilford Press.

Friedlander, M. L. (2015). Use of relational strategies to repair alliance ruptures: How responsive supervisors train responsive psychotherapists. Psychotherapy, 52, 174-179. http://dx.doi.org/10.1037/a0037044

Gergen, K. (1997). El Surgimiento de la Cultura Posmoderna. En K.Gergen (Editor). El Yo Saturado (pp. 150-182). Barcelona: Paidós.

Grant, J., Schofield, M. J., \& Crawford, S. (2012). Managing difficulties in supervision: Supervisors' perspectives. Journal of Counseling Psychology, 59, 528-541. http://dx.doi.org/10.1037/a0030000 
Hawkins, P. (1985). Humanistic psychotherapy supervision: A conceptual framework. Self and Society: Journal of Humanistic Psychology, 13 (2): 69-79. https://doi.org/10.1080/03060497.1985.11084674

Hawkins, P. \& McMahon, A. (2020). Supervision in the Helping Professions (5 $5^{\text {th }}$ Edition). Londres: McGraw-Hill Education (Open University Press).

Hawkins, P., \& Shohet, R. (2006). Supervision in the helping professions: An individual, group and organization approach (3rd ed.). Philadelphia, PA: Open University Press.

Hoffman, L. (1996). Una Postura Reflexiva para la Terapia Familiar. En S. McNamee y K. Gergen (Editores). La terapia como construcción social (pp.25-43). Barcelona: Paidós.

Holloway, E. \& Neufeldt, S. (1995). Supervision: Its contributions to treatment efficacy. Journal of Consulting and Clinical Psychology, 63, 207-213. https://psycnet.apa.org/doi/10.1037/0022-

006X.63.2.207

Jones-Smith, E. (2016). Theories of counseling and psychotherapy: An integrative approach (2da.Ed.). California: Sage.

Kaslow, N. J., Rubin, N. J., Forrest, L., Elman, N. S., Van Horne, B. A., Jacobs, S. C., Huprich, S. K., Benton, S. A., Pantesco, V. F., Dollinger, S. J., Grus, C. L., Behnke, S. H., Miller, D. S. S., Shealy, C. N., Mintz, L. B., Schwartz-Mette, R., Van Sickle, K., \& Thorn, B. E. (2007). Recognizing, assessing, and intervening with problems of professional competence. Professional Psychology: Research and Practice, 38(5), 479492. https://doi.org/10.1037/0735-7028.38.5.479

Ladany, N., Friedlander, M. L., \& Nelson, M. L. (2005). Critical events in psychotherapy supervision: An interpersonal approach. Washington, D.C: American Psychological Association.

Ladany, N., Friedlander, M. L., \& Nelson, M. L. (2016). Supervision Essentials for the Critical Events in Psychotherapy Supervision Model. Washington, D.C: American Psychological Association. (Edición Kindle) http://dx.doi.org/10.1037/14916-000

Ladany, N., Mori, Y. \& Mehr, K. (2013). Effective and ineffective supervision. The Counseling Psychologist, 41 (1), 28-47. https://doi.org/10.1177\%2F0011000012442648

Liese, B. S., \& Beck, J. S. (1997). Cognitive therapy supervision. En C. E. Watkins (Ed.), Handbook of psychotherapy supervision (pp. 114-133). Nueva York: Wiley.
Loganbill, C., Hardy, E., \& Delworth, U. (1982). Supervision: A Conceptual Model. The Counseling Psychologist, $\quad$ 10(1), 3-42. https://doi.org/10.1177/0011000082101002

McNamee, S. \& Gergen, J.K. (1999). Relational Responsibility: Resources for Sustainable Dialogue. California: Sage Publications.

McNeill, B. \& Stoltenberg, C. (2016). Supervision Essentials for the Integrative Developmental Model. Estados Unidos: American Psychological Association.

Miller, S., Duncan, B. \& Hubble, M. (2004). Beyond Integration: The Triumph of Outcome Over Process in Clinical Practice. Psychotherapy in Australia, 10, 2, 2-19. https://scottdmiller.com/wpcontent/uploads/documents/BeyondIntegration.pdf

Milne, D. (2007). An empirical definition of clinical supervision. British Journal of Clinical Psychology, 46 , 437-447. https://doi.org/10.1348/014466507X197415

Milne, D., Aylott, H., Fitzpatrick, H., \& Ellis, M. V. (2008). How does clinical supervision work? Using a "best evidence synthesis" approach to construct a basic model of supervision. The Clinical Supervisor, 27(2),

170-190. https://psycnet.apa.org/doi/10.1080/073252208024 87915

Milne, D., \& Reiser, R. P. (2012). A rationale for evidence-based clinical supervision. Journal of Contemporary Psychotherapy: On the Cutting Edge of Modern Developments in Psychotherapy, 42(3), 139-149. https://doi.org/10.1007/s10879-0119199-8

Monk, G., \& Gehart, D. R. (2003). Sociopolitical activist or conversational partner? Distinguishing the position of the therapist in narrative and collaborative therapies. Family process, 42(1), 19$30 . \quad$ https://doi.org/10.1111/j.15455300.2003.00019.x

Morgan, M. M., \& Sprenkle, D. H. (2007). Toward a common-factors approach to supervision. Journal of Marital \& Family Therapy, 33, 1-17. https://doi.org/10.1111/j.1752-0606.2007.00001.x

Newman, C. F. (2010). Competency in conducting cognitive-behavioral therapy: Foundational, functional, and supervisory aspects. Professional Psychology, Research and Practice, 47(1), 12-19. https://psycnet.apa.org/doi/10.1037/a0018849

Norcross, J. \& Popple, L. (2017). Supervision Essentials for Integrative Psychotherapy. Estados Unidos: American Psychological Association. 
Norcross, J.C. \& Wampold, B.E. (2018). A new therapy for each patient: Evidence-based relationships and responsiveness. Journal of Clinical Psychology, 1-18. https://doi.org/10.1002/jclp.22678

O'Donovan, A., Halford, W.K. \& Walters, B. (2011), Towards Best Practice Supervision of Clinical Psychology Trainees. Australian Psychologist, 46: 101-112. https://doi.org/10.1111/j.17429544.2011.00033.x

Pack, M. (2009). Supervision as a liminal space: Towards a dialogic relationship. Gestalt Journal of Australia and New Zealand, 5, 60-78. https://core.ac.uk/download/pdf/41336304.pdf

Pearson, Q.M. (2006). Psychotherapy-driven supervision: Integrating counseling theories into role-based supervision. Journal of Mental Health Counseling, 28 (3), p241-252. https://psycnet.apa.org/doi/10.17744/mehc.28.3.be 1106w7yg3wvt1w

Pretorius, W. M. (2006). Cognitive-behavioural therapy supervision: Recommended practice. Behavioural \& Cognitive Psychotherapy, 34, 413420.

https://psycnet.apa.org/doi/10.1017/S1352465806 002876

Reiser, R. P., \& Milne, D. (2012). Supervising cognitive-behavioral psychotherapy: Pressing needs, impressing possibilities. Journal of Contemporary Psychotherapy, 42, 161-171. https://psycnet.apa.org/doi/10.1007/s10879-0119200-6

Rigazio-DiGilio, S. (2014). Supervising Couple and Family Therapy practitioners. En Watkins, C.E. Jr. and Milne, D. (Eds). The Wiley international handbook of clinical supervision (pp. 204237). Reino Unido: John Wiley \& Sons, Ltd.

Roth, A.D. \& Pilling, S. (2015). A competence framework for the supervision of psychological therapies. Research Department of Clinical, Educational and Health Psychology. https://www.ucl.ac.uk/pals/sites/pals/files/backgrou nd_document_supervision_competences_july_201 5.pdf

Salvador, J. T. (2016). Integrated Development Model (IMD): A Systematic Review and Reflection. European Scientific Journal, ESJ, 12(19), https://doi.org/10.19044/esj.2016.v12n19p244

Sarnat, J. E. (2010). Key competencies of the psychodynamic psychotherapist and how to teach them in supervision. Psychotherapy: Theory, Research, Practice, Training, 47, 20-27. https://psycnet.apa.org/doi/10.1037/a0018846

Schwartz, H. (2015). International Psychoanalytical Association: Variations on the Eitingon Training Model.

https://www.ipa.world/IPA/iParts/Common/Content Block/PostDefault.aspx?iUniformKey=44e29c575eb0-4c26-83456382aa2bbb3a\&ReplyOptions=fa238730-e491477c-a6e8-d52f298aa9dc\&TagKeys=44936ae9ad33-49d6-8384-

25e7e19a90e4\&DefaultHtmlSessionKey=910b11e 8-f825-472f-a4da-9b7b6f5d8f50\&WebsiteKey=

Simon, G. (2010). Self-supervision, surveillance and transgression. Journal of Family Therapy, 32, pp. 308-325. https://doi.org/10.1111/j.14676427.2010.00505.x

Skovholt, T.M, \& Rønnestad, M.H. (2003). Struggles of the novice counselor and therapist. Journal of Career Development, 30(1), 4558. https://doi.org/10.1177\%2F0894845303030001 03

Stoltenberg, C., Bailey, K. C., Cruzan, C.B., Hart, J. T. \& Ukuku, U. (2014). The Integrative Developmental Model of Supervision. En Watkins, C.E. Jr. and Milne, D. (Eds). The Wiley international handbook of clinical supervision (pp. 576-597). Reino Unido: John Wiley \& Sons, Ltd.

Tarragona, M. (1999). La Supervisión desde una Postura Posmoderna. Psicología Iberoamericana, Vol,7, pp. 68-76.

Todd, T. \& Storm, C. (2014). The Complete Systemic Supervisor: Context, philosophy and pragmatics (2a. Ed). Estados Unidos: Wiley- Blackwell.

Vethencourt D'Escrivan, V. (2018). Cómo vivencian los psicoterapeutas en formación el inicio de su entrenamiento y la supervisión. Psicología Iberoamericana, 26(1), 64-72. https://psicologiaiberoamericana.ibero.mx/index.ph p/psicologia/article/view/34

Villafuerte, A.L.(2016). La Práctica de la Supervisión Clínica en México: Una Actividad Profesional en Desarrollo. Multidisciplinary Health Research, 1(2), 43-46. https://doi.org/10.19136/mhr.a1n2.1560

Watkins, C. E. (1995). Psychotherapy supervision in the 1990s: Some observations and reflections. American Journal of Psychotherapy, 49, 568-581.

Watkins, C.E. (2020). Psychotherapy supervision: an ever-evolving signature pedagogy. World 
Psychyatry, 19-2 (Junio), 244-245. https://doi.org/10.1002/wps.20747

Watkins, E., \& Milne, D. (2014). The Wiley International handbook of clinical supervision. Reino Unido: John Wiley \& Sons, Ltd.

Wheeler, S. \& Richards, K. (2007). The impact of clinical supervision on counsellors and therapists, their practice and their clients. A systematic review of the literature. Counselling and Psychotherapy Research, 7, https://doi.org/10.1080/14733140601185274

White, M. (1997). Supervision as Re-authoring Conversation. En M. White (Editor). Narratives of Therapists' Lives (pp.148-241). Adelaide: Dulwich Center Publications.

Recepción: 02/10/2020

Aceptado: 14/12/2020 